\title{
Energy Harvesting Using an Analog Circuit under Multimodal Vibration
}

\author{
Shigeru Shimose, ${ }^{1}$ Kanjuro Makihara, ${ }^{2}$ and Junjiro Onoda ${ }^{1}$ \\ ${ }^{1}$ Institute of Space and Astronautical Science (ISAS), Japan Aerospace Exploration Agency (JAXA), \\ 3-1-1 Yoshinodai, Chuo Ward, Sagamihara, Kanagawa 252-5210, Japan \\ ${ }^{2}$ Department of Aerospace Engineering, Tohoku University, 6-6-01 Aoba, Aramaki, Aoba Ward, Sendai, Miyagi 980-8579, Japan
}

Correspondence should be addressed to Shigeru Shimose, shimose.shigeru@jaxa.jp

Received 7 October 2012; Accepted 26 December 2012

Academic Editor: Xiaoning Jiang

Copyright (C) 2013 Shigeru Shimose et al. This is an open access article distributed under the Creative Commons Attribution License, which permits unrestricted use, distribution, and reproduction in any medium, provided the original work is properly cited.

The efficiency of harvesting energy from a vibrating structure using a piezoelectric transducer and a simple analog circuit is investigated experimentally. This analog circuit was originally invented for a synchronized switch damping on inductor (SSDI) technique, which enhances the damping of mechanical vibration. In this study, the circuit is used to implement a synchronized switch harvesting on inductor (SSHI) technique. A multiple degree of freedom (MDOF) structure is excited by single sinusoidal forces at its resonant frequencies and by random forces. The piezoelectric transducer converts this mechanical energy into electrical energy which is harvested using a standard rectifier bridge circuit with and without our analog circuit. Experimental results show that our analog circuit makes it possible to harvest twice as much energy under both single sinusoidal and random vibration excitations.

\section{Introduction}

Energy harvesting techniques have been studied extensively in recent years. Energy harvesting is a process by which energy is captured and stored. Energy can be harvested from various power sources, including wind power, solar power, ocean tides, heart, magnetic fields, and structural vibrations. We focused on the vibration energy of a structure, using the piezoelectric effect to convert structural vibration energy into electrical energy. There is substantial research on this technique, as reviewed by Sodano et al. [1]. Lesieutre et al. [2] addressed the damping associated with energy harvesting from structural vibrations.

Badel et al. [3-5] proposed a synchronized switch harvesting on inductor (SSHI) technique to improve energy harvesting. SSHI is based on vibration suppression technique named synchronized switch damping on inductor (SSDI). Both SSHI and SSDI use a piezoelectric transducer attached to the structure and connected to an inductive circuit having an on-off switch [6-10]. The switch in the circuit is flipped at each extremum of displacement of the structure. A displacement sensor and a controller are needed to synchronize the switching commands with the mechanical vibration. In a self-powered system, these sensors and controllers need to be driven using a fraction of the harvested energy.

We previously invented an analog circuit that automatically performs switching without an external energy source [11]. We describe in this paper how this analog circuit enhances the energy harvesting performance when used with SSHI.

Although many studies [12-14] have been conducted on SSHI, most of them are limited to the sinusoidal vibration of a single degree of freedom (SDOF) structure. This paper focuses on the energy harvesting performance of our analog vibration suppression system for a multiple degree of freedom (MDOF) structure under various excitations. First, we describe the SSDI mechanism. Next, we describe our analog self-powered device. Finally, we present the experimental 


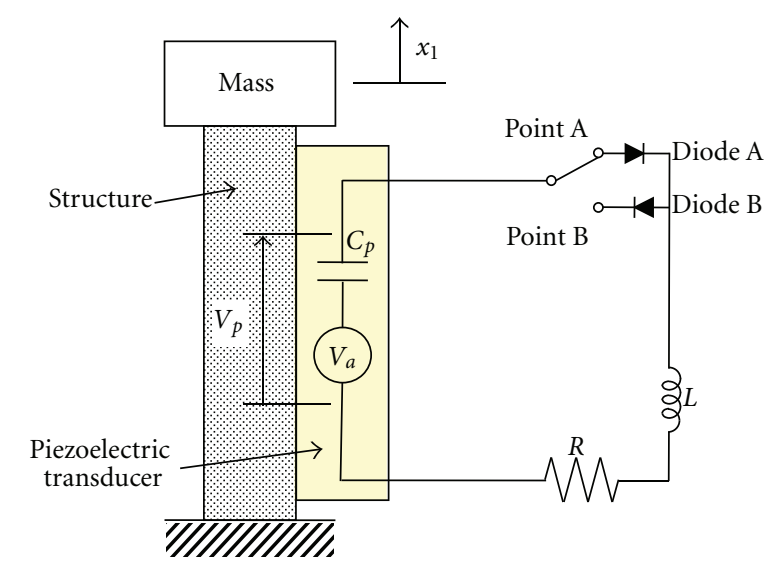

FIGURE 1: SDOF vibration suppression system with original SSDI.

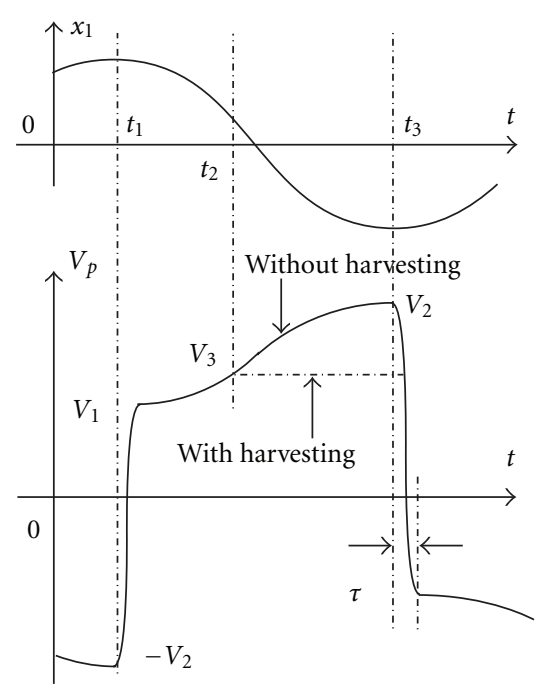

FIGURE 2: Schematic illustration of piezoelectric voltage with switching inductive circuit.

results demonstrating the energy harvesting performance of our device for an MDOF structure under sinusoidal and random vibration excitations.

\section{SSDI and SSHI Systems}

2.1. Original SSDI Technique. The SSDI mechanism is described in [6-10]. For simplicity, we consider the SDOF system shown in Figure 1. The system is composed of a mass, a structure, a piezoelectric transducer attached to the structure, and a switchable inductive circuit. The piezoelectric transducer is modeled by a voltage generator $V_{a}$ and a capacitor $C_{P}$.

As shown in $[8,10,15]$, the basic concept of the SSDI technique is to flip the switch from point B to point A at the moment when displacement $x_{1}$ or voltage $V_{P}$ reaches a maximum, as well as to flip the switch from point $\mathrm{A}$ to point B when $x_{1}$ or $V_{P}$ reaches a minimum. Figure 2 shows the vibration in $V_{P}$. When the displacement of mass reaches

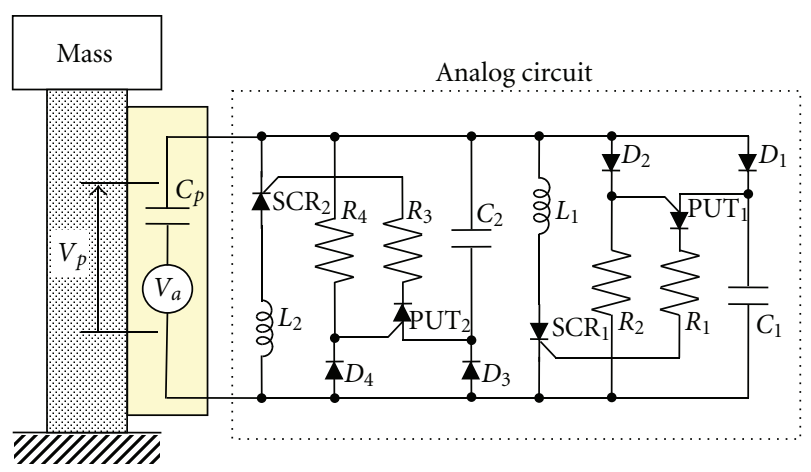

FIGURE 3: SDOF vibration suppression system with our analog circuit.

TABLE 1: Device parameters of our analog circuit.

\begin{tabular}{lc}
\hline$C_{P}$ & $0.51 \mu \mathrm{F}$ \\
$C_{1}, C_{2}$ & $0.1 \mu \mathrm{F}$ \\
$R_{1}, R_{3}$ & $100 \Omega$ \\
$R_{2}, R_{4}$ & $100 \mathrm{M} \Omega$ \\
$L_{1}, L_{2}$ & $20 \mathrm{mH}$ \\
\hline
\end{tabular}

a maximum at $t=t_{1}$, the switch is flipped from point A to point $B$. Current starts flowing in the inductive circuit shown in Figure 1 and stops when the voltage reaches a positive value $V_{1}$, owing to the inductor and diode $\mathrm{B}$ in the circuit. During the subsequent half cycle of mechanical vibration, as the displacement of mass reaches the minimum value, voltage $V_{P}$ changes from $V_{1}$ to $V_{2}$. At time $t=t_{3}$, the position of the switch is flipped from point $\mathrm{B}$ to point $\mathrm{A}$, and the piezoelectric voltage changes from $V_{2}$ to $-V_{1}$.

This switching technique stores the electric charge in the piezoelectric transducer rather than allowing it to be dissipated, increasing the magnitude of $V_{P}$ and thus increasing the force generated by the piezoelectric transducer to suppress the vibration. Furthermore, this switching inverses the polarity of $V_{P}$ in synchronism with the mechanical vibration so that the vibration is effectively suppressed.

2.2. Analog Self-Powered SSDI. The original SSDI $[8,10$, 15] needs external power for measurement, calculation, and switching. To eliminate the external power supply, we have invented a simple passive analog circuit that performs SSDI switching automatically, as shown in Table 1 and Figure 3.

Figure 3 shows our electric circuit for SSDI. When the voltage across the piezoelectric transducer $V_{P}$ passes the maximum and starts to decrease, the anode voltage of the programmable unijunction transistor $\mathrm{PUT}_{1}$ is maintained at the peak by diode $D_{1}$ and capacitor $C_{1}$. However, the gate voltage of $\mathrm{PUT}_{1}$ decreases following the decrease in $V_{P}$ due to mechanical vibration. Therefore, $\mathrm{PUT}_{1}$ is turned on and the charge stored in $C_{1}$ flows through thyristor $\mathrm{SCR}_{1}$ as its gate current. The gate current turns $\mathrm{SCR}_{1}$ on, and the charge stored in the capacitor of the piezoelectric transducer, $C_{P}$, flows through the inductor. Because the thyristor prevents the electric current from flowing in the opposite direction, 


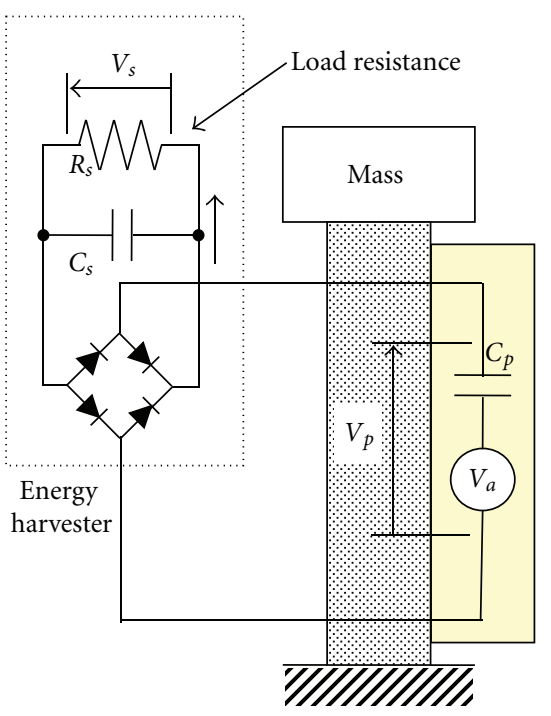

(a)

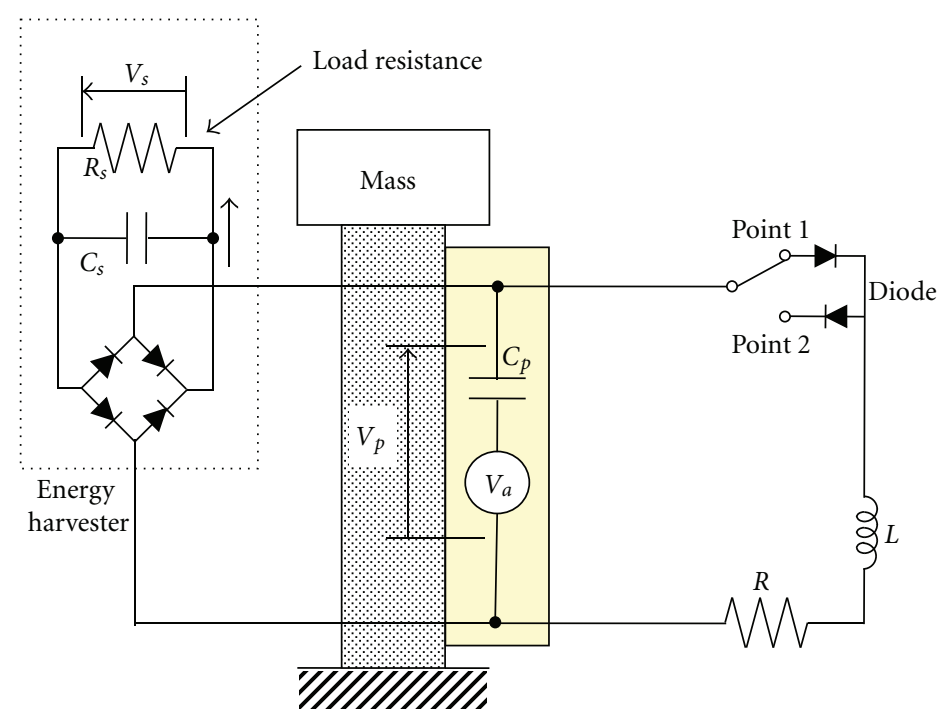

(b)

FIGURE 4: (a) SDOF energy harvester connected a piezoelectric transducer. (b) SDOF energy harvester connected a piezoelectric transducer with original SSDI.

turning the thyristor on is equivalent to flipping the switch in Figure 1 to point $\mathrm{A}$. When the value of $V_{P}$ reaches a minimum and starts to increase, $\mathrm{PUT}_{2}$ is similarly turned on, and subsequently, $\mathrm{SCR}_{2}$ is also turned on. This action is equivalent to flipping the switch in Figure 1 to point $\mathrm{B}$.

In effect, this analog circuit flips the switch in Figure 1 to point A when $V_{P}$ is approximately at a maximum and flips the switch to point $\mathrm{B}$ when $V_{P}$ is approximately at a minimum. Therefore, this simple analog circuit performs the functions of a sensor, a controller, and a switch for SSDI.

2.3. Energy Harvesting with Original SSHI. An electric circuit consisting of a piezoelectric transducer connected to an energy harvester and a load resistance $R_{S}$ is shown in Figure 4(a). The energy harvester is composed of four diodes and a capacitor $C_{S}$. This is a basic energy harvesting system with a piezoelectric transducer. A bridge circuit with four diodes rectifies the electrical energy from the piezoelectric transducer and stores it in capacitor $C_{S}$.

Figure 4(b) shows a typical system for energy harvesting with SSHI. As shown in this figure, a switchable inductive circuit is connected to the system shown in Figure 4(a). SSHI tunes the switch in Figure 4(b) just as SSDI does. Because this switching increases the absolute value of $V_{P}$, as mentioned, SSHI can effectively harvest energy. In Figure 2, the broken line shows the vibration in $V_{P}$ when some energy is harvested by SSHI, whereas the solid line shows the variation in $V_{P}$ when SSDI is used. The figure shows that when the absolute value of $V_{P}$ reaches the voltage of $C_{S}$, the energy starts to flow into $C_{S} . C_{S}$ has a large capacitance, causing $V_{P}$ to plateau.

2.4. Energy Harvesting with Analog Self-Powered SSDI. The SSDI technique is implemented as shown in Figure 5, by replacing the switchable inductive shunt circuit shown in
Figure 1 with our analog circuit. Similarly, SSHI is implemented as shown in Figure 5 by replacing the switchable shunt circuit in Figure 4(b) by our analog circuit.

\section{Experiment}

3.1. Experimental Setup. Figures 6 and 7 show a view of the experimental system, which consists of two masses, a pantograph-type displacement-magnification mechanism, a piezoelectric transducer (PSt 1000/10/200-VS18, Piezomechanik $\mathrm{GmbH}$ ), two cantilevered beams, a spring, a vibration shaker, and a platform. The displacement-magnification mechanism is attached to the upper beam, and upper side of the platform is used to accommodate the small elongation of the piezoelectric transducer in response to the large amplitude of vibration. The natural frequencies of the first and second vibration modes at a constant electric charge are 20.3 and $36.6 \mathrm{~Hz}$, respectively [16].

The experiment is performed under three types of vibration excitations: a sinusoidal vibration excitation at the first mode resonant frequency, a sinusoidal vibration excitation at the second mode resonant frequency, and a random vibration excitation. We investigated the performance in harvesting energy using our system shown in Figure 5 for various values of load resistance $R_{S}$.

3.2. Experimental Results with Sinusoidal Excitation. Figure 8 (a) shows the steady-state variations in displacement of the upper mass 1 , the voltage of the piezoelectric transducer $V_{P}$, and the harvested voltage $V_{S}$ under a sinusoidal excitation at the first mode resonant frequency. In this case, the value of $R_{S}$ is very large. At each extremum of displacement, the polarity of voltage $V_{P}$ reverses. This voltage behavior is a typical feature of SSHI. Figure 8(b) shows a magnified view of Figure $8(\mathrm{a})$. When the absolute value of $V_{P}$ is increased 


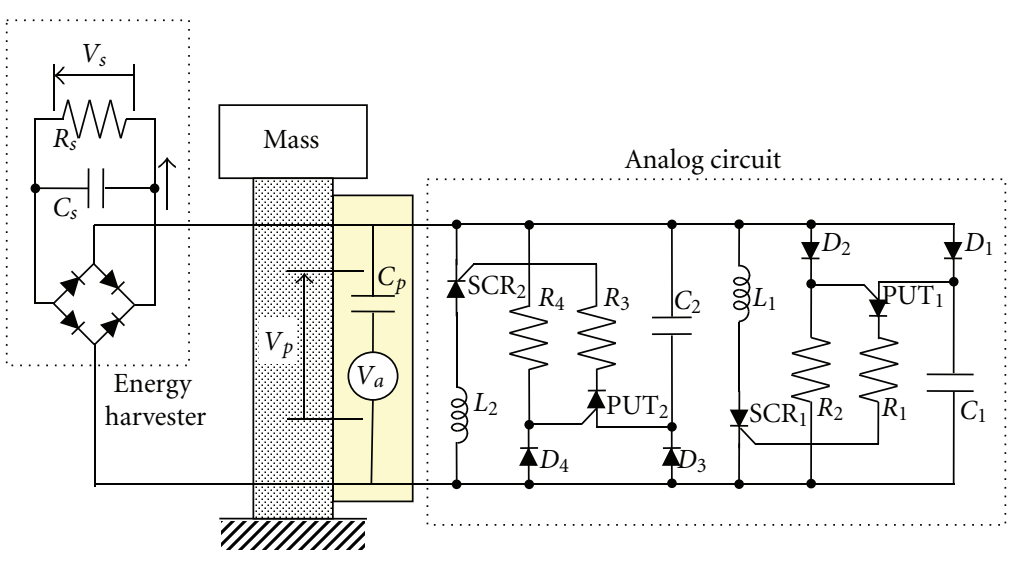

FIGURE 5: SDOF energy harvester with our analog self-powered circuit.

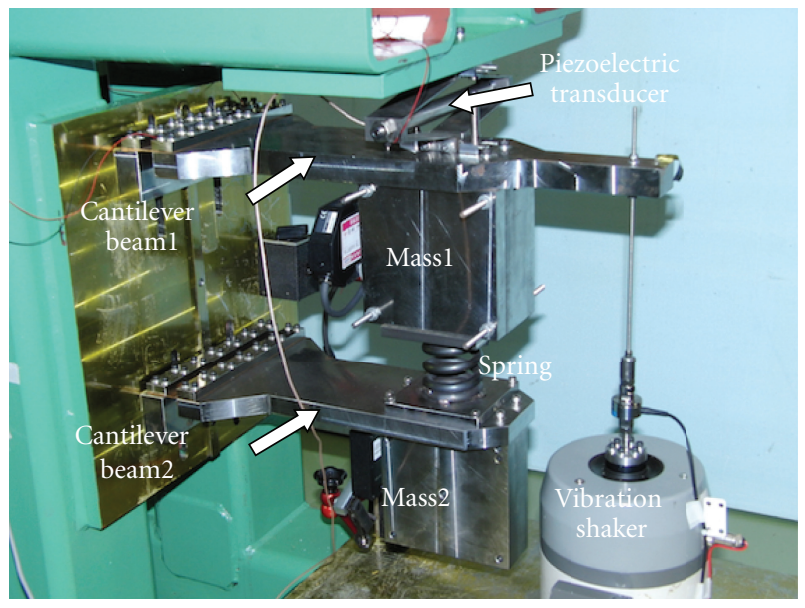

FIGURE 6: Mechanical part in 2-DOF system.

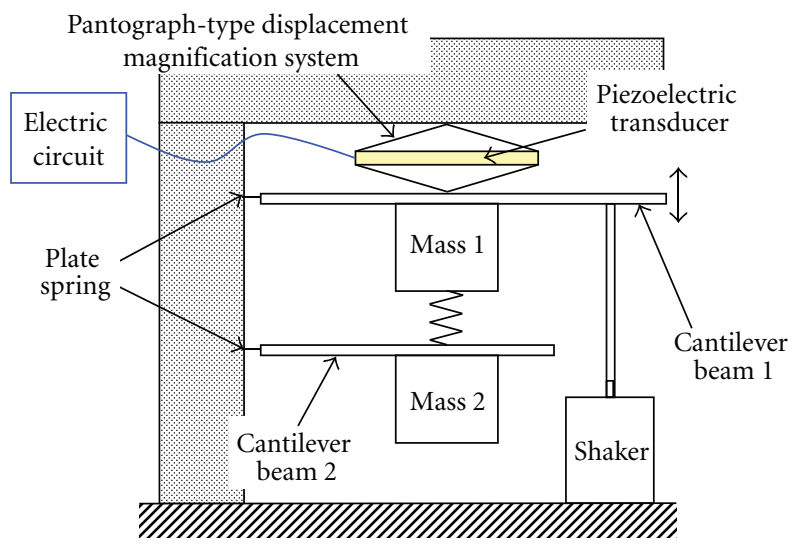

FIGURE 7: Mechanical experimental configuration in 2-DOF system.

to a certain value, energy starts flowing into capacitor $C_{S}$, and as a result, $V_{P}$ stays almost constant, making a plateau in the waveform. The difference between the plateau voltage and voltage $V_{S}$ is attributed to the forward voltage of the diodes in the rectifier circuit. Figure 8(b) shows that the difference is $1.2 \mathrm{~V}$, which is consistent with the forward voltage of $0.6 \mathrm{~V}$ for each diode.

Figure 9(a) shows a comparison of the normalized harvested energy for various values of load resistance under a sinusoidal excitation at the first and second mode resonant frequencies. The energy dissipated by the load resistance is influenced by the value of resistance $R_{S}$. To optimize the harvested power, we apply various load resistances in the electric circuit. The horizontal axis represents the load resistance, and the vertical axis represents the harvested power divided by the mean square of displacement of mass 1 , with and without our analog circuit. These figures indicate that our system significantly increases the energy harvested from the MDOF structure under sinusoidal vibrations. Although the amount of energy harvested depends on the value of the electric load $R_{S}$, the amount is drastically increased using our circuit under vibration excitation at both the first and the second mode resonant frequencies.

3.3. Experimental Results with Random Excitation. We also carried out experiments using random excitation. The vibration shaker connected the upper cantilever through the load cell. A function generator creates an input voltage wave and sends it to vibration shaker. The PSD of the random excitation force is constant over the frequency range from $10 \mathrm{~Hz}$ to $50 \mathrm{~Hz}$.

Figure 9(b) shows the normalized harvested energy for various values of load resistances under random excitation. The horizontal axis is the load resistance, and the vertical axis is the harvested power divided by the mean square of displacement of mass 1 , with and without our analog circuit. This figure indicates that our system significantly increases the energy harvested from the MDOF structure under random vibrations. Although the amount of energy harvested depends on the value of the electric load $R_{S}$, the amount is drastically increased using our circuit under random excitation.

The harvested energy can be increased by a factor of at least 2.3 using our simple analog circuit not only under sinusoidal vibration excitation but also under random vibration excitation. 


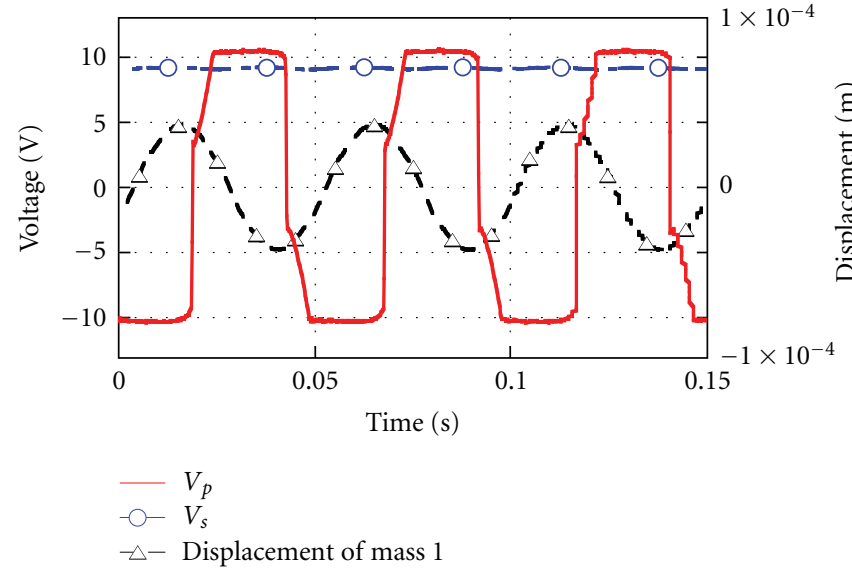

(a)

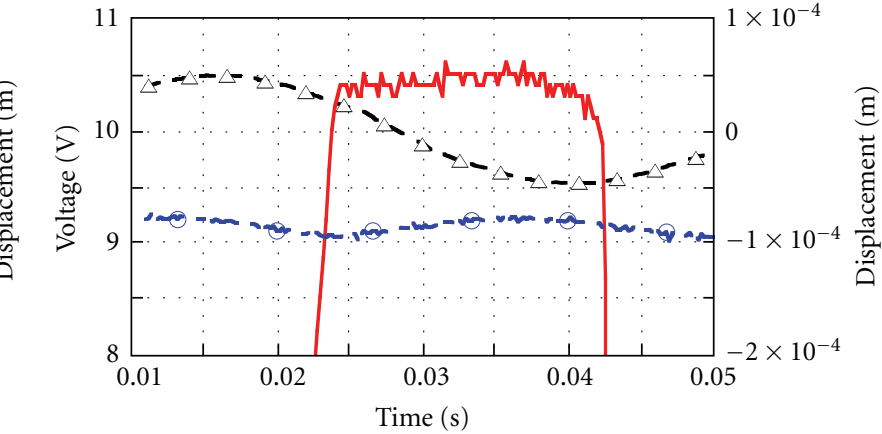

$-V_{p}$
$-V_{s}$
$-\triangle-$ Displacement of mass 1

(b)

FIGURE 8: (a) Time history of the piezoelectric voltage, harvested voltage, and displacement of mass 1 under first mode sinusoidal vibration excitation $\left(C_{S}=47 \mathrm{mF}, R_{S}=\infty \Omega\right)$. (b) Magnified view of (a).

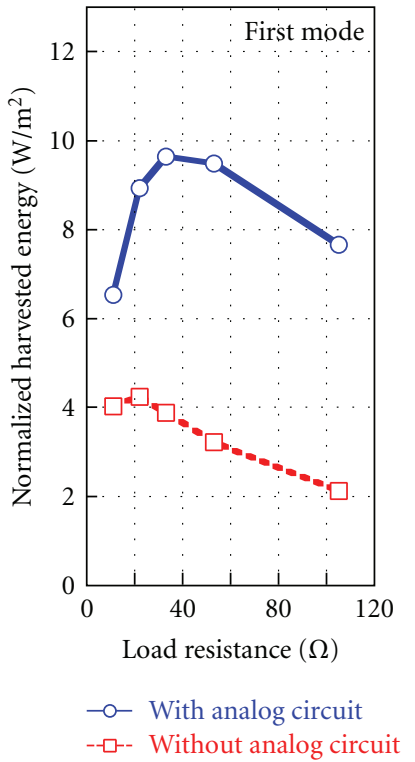

(a)
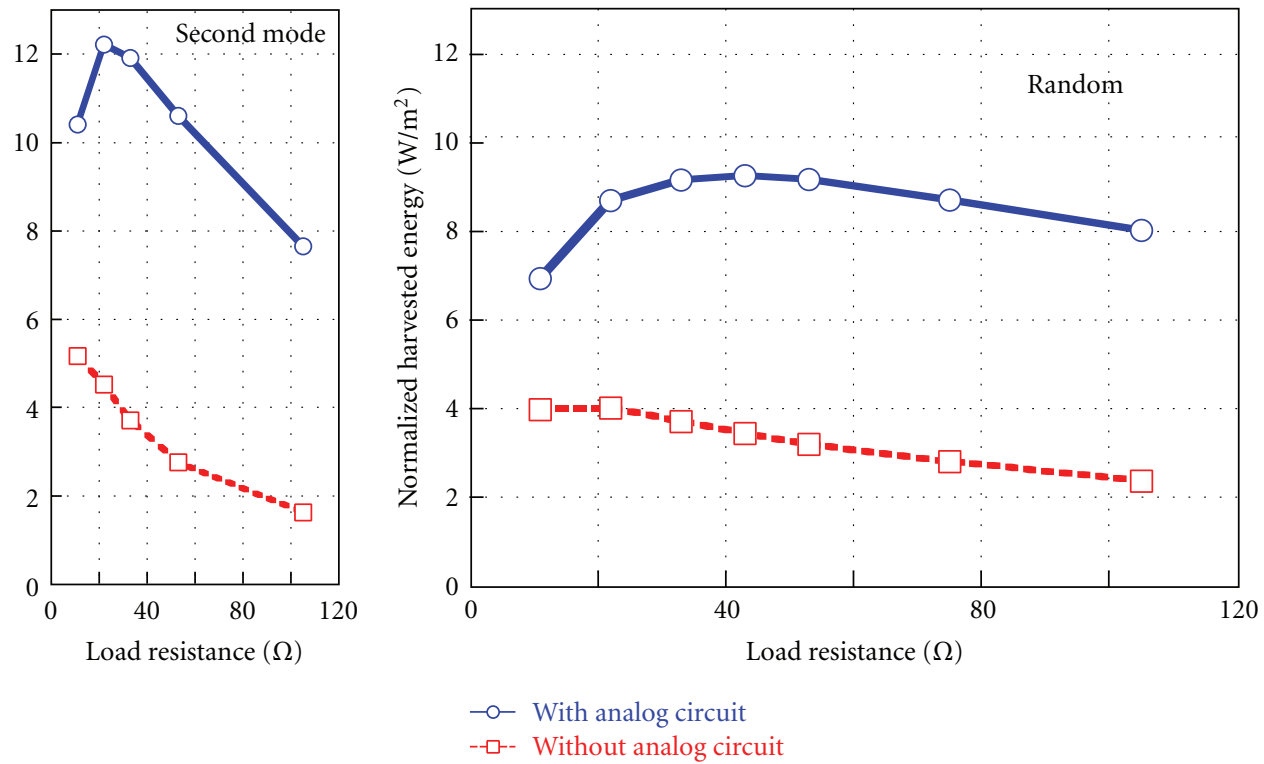

(b)

FIGURE 9: (a) Comparison of normalized harvest power for various load resistances under sinusoidal vibration excitations. (b) Comparison of normalized harvest power for various load resistances under random vibration excitations.

\section{Conclusion}

We proposed a simple analog circuit to implement SSHI for harvesting energy from structural vibrations using piezoelectric transducers, and we investigated the resulting performance. Experiments were performed using an MDOF structure under sinusoidal vibration excitation at resonance frequencies and under random vibration excitation. Experimental results show that connecting our simple analog circuit to the standard circuit more than doubles the amount of energy harvested under both single sinusoidal vibration excitation and random vibration excitation.

\section{Acknowledgment}

This research was supported by a Grant-in-Aid for Scientific Research (B) (no. 20360386) from the Japan Society for Promotion of Science.

\section{References}

[1] H. A. Sodano, D. J. Inman, and G. Park, "A review of power harvesting from vibration using piezoelectric materials," Shock and Vibration Digest, vol. 36, no. 3, pp. 197-205, 2004.

[2] G. A. Lesieutre, G. K. Ottman, and H. F. Hofmann, "Damping as a result of piezoelectric energy harvesting," Journal of Sound and Vibration, vol. 269, pp. 991-1002, 2003. 
[3] A. Badel, D. Guyomar, E. Lefeuvre, and C. Richard, "Efficiency enhancement of a piezoelectric energy harvesting device in pulsed operation by synchronous charge inversion," Journal of Intelligent Material Systems and Structures, vol. 16, no. 10, pp. 889-901, 2005.

[4] D. Guyomar, C. Magnet, E. Lefeuvre, and C. Richard, "Power capability enhancement of a piezoelectric transformer," Smart Materials and Structures, vol. 15, no. 2, pp. 571-580, 2006.

[5] K. Makihara, J. Onoda, and T. Miyakawa, "Low energy dissipation electric circuit for energy harvesting," Smart Materials and Structures, vol. 15, no. 5, pp. 1493-1498, 2006.

[6] W. W. Clark, "Vibration control with state-switched piezoelectric materials," Journal of Intelligent Material Systems and Structures, vol. 11, no. 4, pp. 263-271, 2000.

[7] C. Richard, D. Guyomar, D. Audigier, and G. Ching, "Semipassive damping using continuous switching of a piezoelectric device," in Smart Structures and Materials-Passive Damping and Isolation, vol. 3672 of Proceedings of SPIE, pp. 104-111, March 1999.

[8] C. Richard, D. Guyomar, D. Audigier, and H. Bassaler, "Enhanced semi passive damping using continuous switching of a piezoelectric device on an inductor," in Smart Structures and Materials 2000: Damping and Isolation, vol. 3989 of Proceedings of SPIE, pp. 288-299, March 2000.

[9] L. R. Corr and W. W. Clark, "Comparison of low-frequency piezoelectric switching shunt techniques for structural damping," Smart Materials and Structures, vol. 11, no. 3, pp. 370-376, 2002.

[10] J. Onoda, K. Makihara, and K. Minesugi, "Energy-recycling semi-active method for vibration suppression with piezoelectric transducers," AIAA Journal, vol. 41, no. 4, pp. 711-719, 2003.

[11] J. Onoda, "Some advances in energy recycling semi-active vibration suppression," Advances in Science and Technology, vol. 56, pp. 345-354, 2008.

[12] E. Lefeuvre, A. Badel, C. Richard, L. Petit, and D. Guyomar, "High efficiency piezoelectric vibration energy reclamation," in International Symposium on Smart Structure and Materials, vol. 5390, pp. 379-387, March 2004.

[13] A. Badel, A. Benayad, E. Lefeuvre, L. Lebrun, C. Richard, and D. Guyomar, "Single crystals and nonlinear process for outstanding vibration-powered electrical generators," IEEE Transactions on Ultrasonics, Ferroelectrics, and Frequency Control, vol. 53, no. 4, pp. 673-684, 2006.

[14] E. Lefeuvre, A. Badel, C. Richard, L. Petit, and D. Guyomar, "A comparison between several vibration-powered piezoelectric generators for standalone systems," Sensors and Actuators A, vol. 126, no. 2, pp. 405-416, 2006.

[15] K. Makihara, J. Onoda, and K. Minesugi, "Comprehensive assessment of semi-active vibration suppression including energy analysis," Journal of Vibration and Acoustics, vol. 129, no. 1, pp. 84-93, 2007.

[16] K. Makihara, S. Takeuchi, S. Shimose, J. Onoda, and K. Minesugi, "Innovative digital self-powered autonomous system for multimodal vibration suppression," AIAA Journal, vol. 50, no. 9, pp. 2004-2011, 2012. 

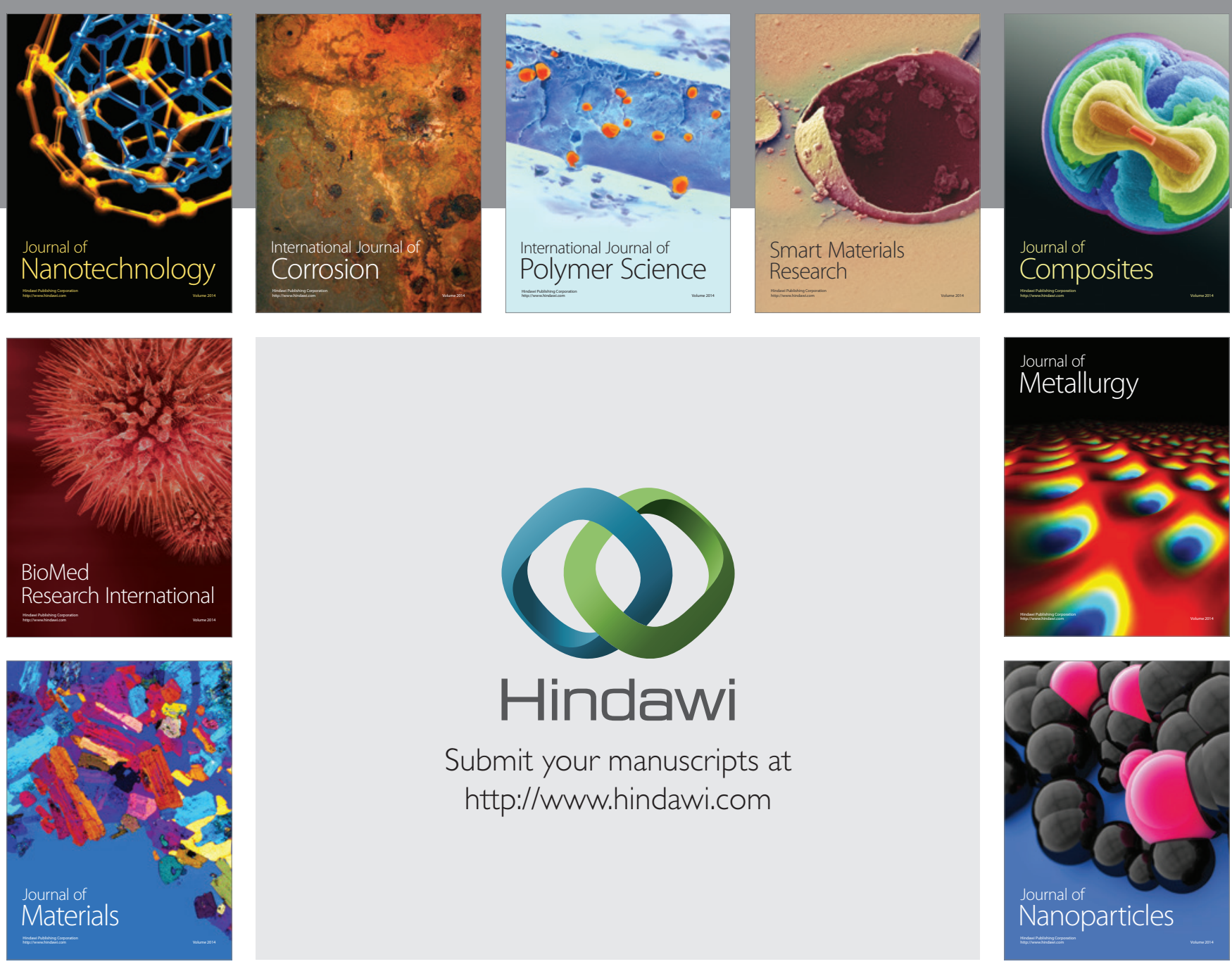

Submit your manuscripts at http://www.hindawi.com
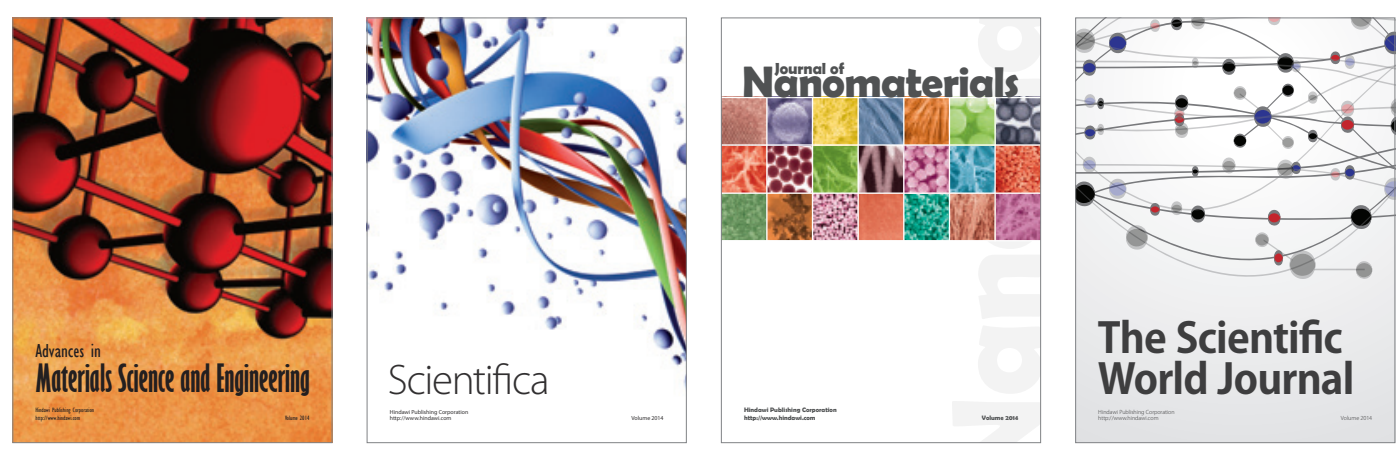

\section{The Scientific World Journal}
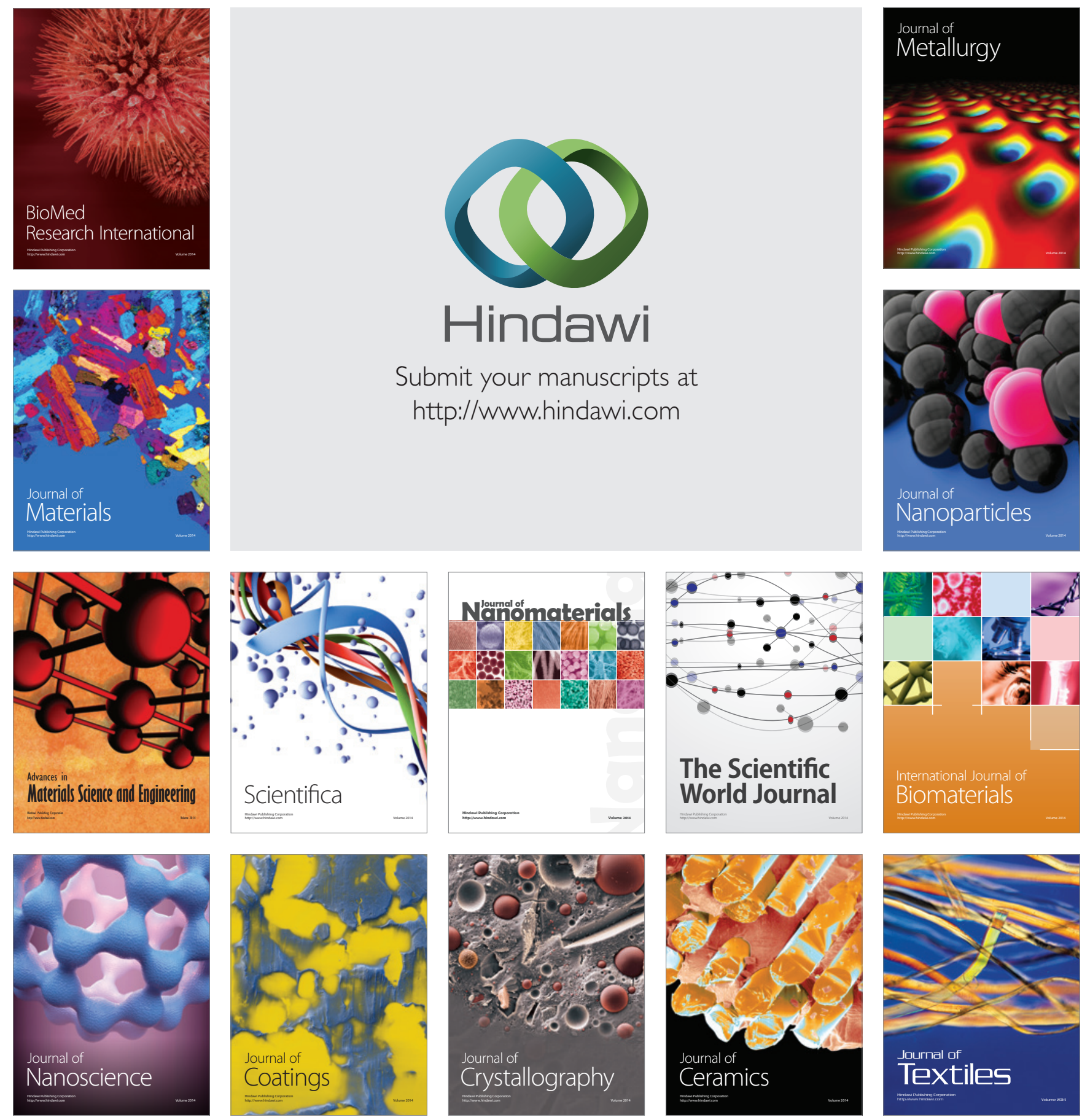